\title{
High Excitation Efficiency of Channel Plasmon Polaritons in Tailored, UV-Lithography-
} Defined V-Grooves

Smith, Cameron; Thilsted, Anil Haraksingh; Garcia-Ortiz, Cesar E.; Radko, I. P.; Marie, Rodolphe ; Jeppesen, Claus; Vannahme, Christoph; Bozhevolnyi, S. I.; Kristensen, Anders

\section{Published in:}

Proceedings of 2014 Conference on Lasers and Electro-Optics (CLEO)

Publication date:

2014

Document Version

Publisher's PDF, also known as Version of record

Link back to DTU Orbit

Citation (APA):

Smith, C., Thilsted, A. H., Garcia-Ortiz, C. E., Radko, I. P., Marie, R., Jeppesen, C., Vannahme, C., Bozhevolnyi, S. I., \& Kristensen, A. (2014). High Excitation Efficiency of Channel Plasmon Polaritons in Tailored, UVLithography-Defined V-Grooves. In Proceedings of 2014 Conference on Lasers and Electro-Optics (CLEO) (pp. 1-2). IEEE.

\section{General rights}

Copyright and moral rights for the publications made accessible in the public portal are retained by the authors and/or other copyright owners and it is a condition of accessing publications that users recognise and abide by the legal requirements associated with these rights.

- Users may download and print one copy of any publication from the public portal for the purpose of private study or research.

- You may not further distribute the material or use it for any profit-making activity or commercial gain

- You may freely distribute the URL identifying the publication in the public portal 


\title{
High Excitation Efficiency of Channel Plasmon Polaritons in Tailored, UV-Lithography-Defined V-Grooves
}

\author{
Cameron L.C. Smith, ${ }^{1}$ Anil H. Thilsted, ${ }^{1}$ Cesar E. Garcia-Ortiz, ${ }^{2}$ Ilya P. Radko, ${ }^{2}$ Rodolphe Marie, ${ }^{1}$ \\ Claus Jeppesen, ${ }^{3}$ Christoph Vannahme, ${ }^{1}$ Sergei I. Bozhevolnyi ${ }^{2}$ and Anders Kristensen ${ }^{1}$ \\ ${ }^{I}$ Department of Micro- and Nanotechnology, Technical University of Denmark, DK-2800 Kongens Lyngby, Denmark \\ ${ }^{2}$ Institute of Technology and Innovation (ITI), University of Southern Denmark, DK-5230 Odense M, Denmark \\ ${ }^{3}$ Department of Photonics Engineering, Technical University of Denmark, DK-2800 Kongens Lyngby, Denmark \\ Corresponding author: cameron.smith@nanotech.dtu.dk; Phone: +45-4525 6331, Fax: +45- 45887762
}

\begin{abstract}
We demonstrate $>50 \%$ conversion of light to V-groove channel plasmon-polaritons (CPPs) via compact waveguide-termination mirrors. Devices are fabricated using UV-lithography and crystallographic silicon etching. The $\mathrm{V}$-shape is tailored by thermal oxidation to support confined CPPs.

(C) 2014 Optical Society of America

OCIS codes: (220.3740) Lithography; (230.7380) Waveguides, channeled; (240.6680) Surface plasmons
\end{abstract}

\section{Introduction}

Waveguide configurations based on surface plasmon polaritons (SPPs) possess unique scaling properties that allow one to concentrate light below the diffraction limit [1]. This feature of SPP-based waveguides has signaled the possibility of developing a new generation of subwavelength-integrated optical waveguides, circuits and devices. Vshaped grooves in metals supporting channel plasmon-polaritons (CPPs) represent a promising plasmonic waveguide configuration by providing a competitive confinement-loss tradeoff in addition to efficient broadband transmission around sharp bends. V-groove-supported CPPs have enabled the demonstration of compact plasmonic circuit components and novel nanofocusing elements [2]. However, the viability of plasmonic V-grooves for significant implementation requires a convenient approach to efficiently excite the CPP modes in a way that can be realized using affordable fabrication techniques.

CPP excitation to-date involves end-fire coupling, requiring a cleaved sample end-facet which may damage the waveguide entrance. This problem applies generally to SPP-based waveguide devices and has motivated the investigation of numerous directional nanoantenna configurations to conveniently launch plasmons via normally incident light, such as Yagi-Uda design, single element, phase-engineered, nanopatch and Bragg resonator type arrangements. Recently, Radko et al. developed a coupling arrangement to excite V-groove-supported CPPs via normally incident illumination by tapering the waveguide terminations to form angled nanomirrors [3]. These nanomirrors launched CPPs into nanoscale V-groove waveguides, yet the maximum in-coupling efficiency was $\sim 10 \%$ and, like the arrangements mentioned above, required expensive and time-consuming fabrication methods such as focused ion beam milling or electron beam lithography.
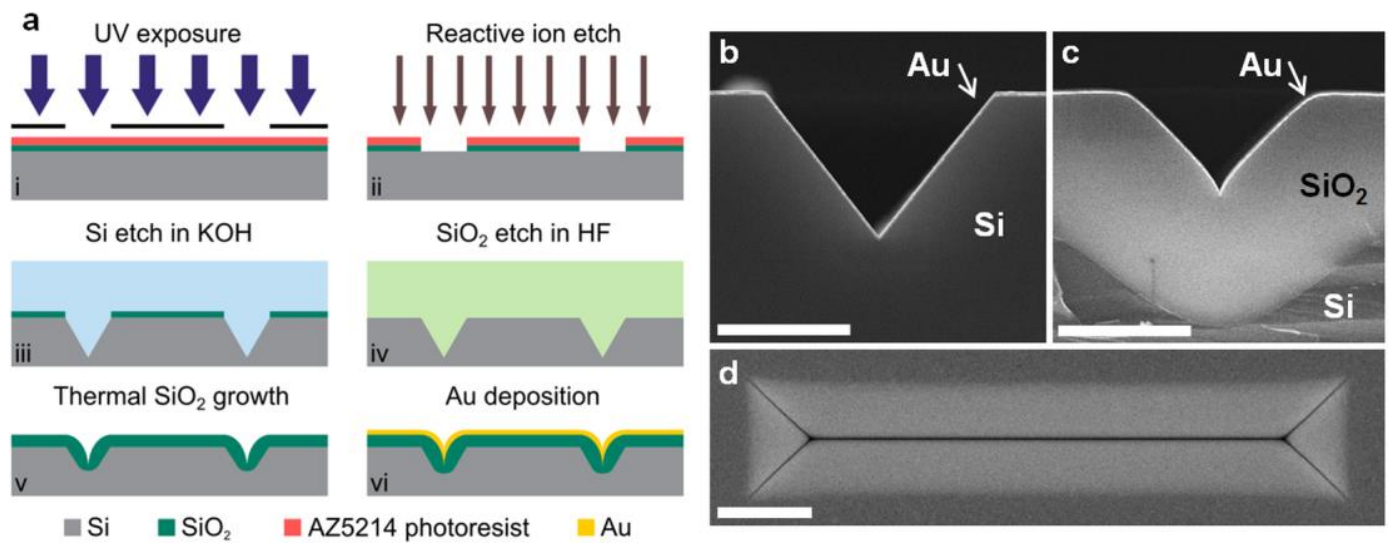

Fig. 1. (a) Fabrication procedure for Au V-grooves and coupling mirrors involving UV-lithography. (b)-(d) SEM images of resulting Vgrooves, scale bars are $2 \mu \mathrm{m}$. (b) V-groove without thermal oxidation. (c) V-groove with a $2320 \mathrm{~nm} \mathrm{SiO}_{2}$ layer. (d) Top view of (c). 
In this work, we experimentally demonstrate the $>50 \%$ in-coupling efficiency of V-groove CPPs using freely propagating light directed at normal incidence onto waveguide-termination mirrors. The $\mathrm{V}$-grooves and mirrors are both defined during the same conventional UV-lithography step which, combined with chemical etching of silicon crystallographic planes [4], forms the smooth, V-shaped profiles innately in a wafer-scale procedure. The silicon Vgrooves are tailored by thermal oxidation of the silicon to sharpen the groove angle and ensure the existence of wellconfined CPP modes. The experimental results are supported by finite element method (FEM) calculations.

\section{Fabrication and Characterization}

The fabrication of our V-grooves and waveguide-termination mirrors is shown in Fig. 1 [5]. The process involves UV-lithography to define the perimeter of the V-groove devices. A crystallographic wet etch of silicon planes forms the smooth V-groove sidewalls and mirrors simultaneously. A thermally grown silicon dioxide $\left(\mathrm{SiO}_{2}\right)$ layer on silicon modifies the V-shape geometry. A $70 \mathrm{~nm}$ gold layer is deposited to form the resulting plasmonic waveguide.

The device design is performed with 2D FEM calculations in COMSOL at a free space wavelength of $\lambda=811 \mathrm{~nm}$ [5]. The magnitude of the electric field distributions for fundamental plasmonic $\mathrm{V}$-groove modes of different cross-sections are plotted in Figs. 2(b)-(c): (b) No oxide layer and (c) a $2320 \mathrm{~nm} \mathrm{SiO}{ }_{2}$ layer. In Fig 2(b) it is evident that only wedge-based modes exist for the case without oxidation, whereas in Fig 2(c) the thermally grown $\mathrm{SiO}_{2}$ layer sharpens the $\mathrm{V}$-shaped profiles and increases the electric field confinement to a clear CPP mode.

To verify the calculations and determine the nature of the propagating modes, we illuminate $\mathrm{V}$-groove waveguides with and without the $\mathrm{SiO}_{2}$ layer, see Figs. 2(d)-(f) [5]. The V-groove device without $\mathrm{SiO}_{2}$ layer (Fig. 2(d) only supports wedge-based modes. The device with $\mathrm{SiO}_{2}$ layer (Fig. 2(e)) shows a clear single intensity peak with an in-coupling efficiency of $>50 \%$. Rotating the polarization $90^{\circ}$ (Fig. 2(f)) results in a factor of 10 reduction in out-coupled light, supporting the assumption that it is a TE-like CPP mode with weak longitudinal component.
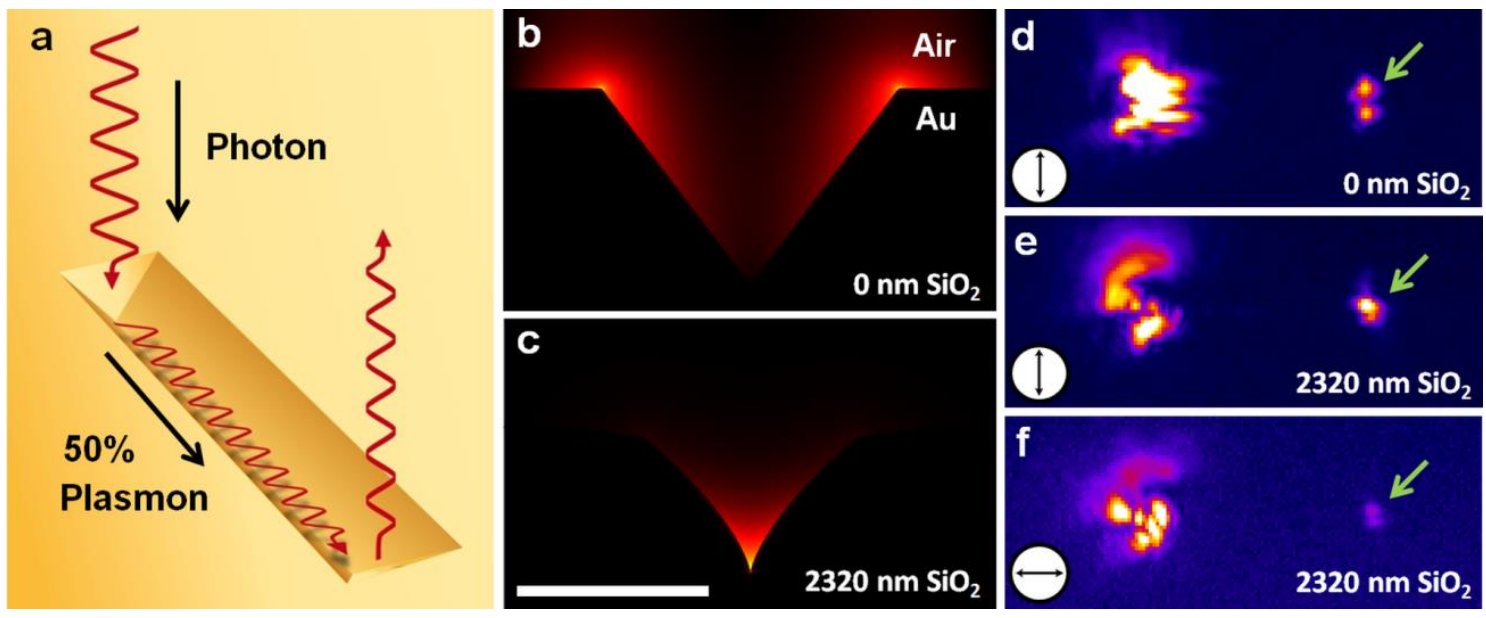

Fig. 2. (a) Illustration of the in- and out-coupling of light in the V-groove devices. (b)-(c) 2D FEM calculations of the electric field for different V-groove cross-sections. Scale bar is $2 \mu \mathrm{m}$. (d)-(f) Experimentally observed radiation from V-groove samples. Circular inset denotes the incident electric field polarization. $\mathrm{SiO}_{2}$ layer modification alters the propagating mode distribution. The polarization dependence indicates a CPP mode.

In summary, we demonstrate the highly efficient ( $>50 \%)$ conversion of freely propagating light to $\mathrm{V}$-groove channel plasmon-polaritons (CPPs) via compact waveguide-termination mirrors. The devices are fabricated using UV-lithography and crystallographic silicon etching, pointing to wafer-scale production. The V-shape is tailored by thermal oxidation to support confined CPPs. The coupling configuration is ideal for efficient excitation of plasmonic elements collinear through a microscope objective, such as for integrated lab-on-a-chip devices, or for facilitating efficient inter- and intra-chip communication in planar or multi-leveled photonic information processing systems.

\section{References}

[1] D. K. Gramotnev and S. I. Bozhevolnyi, "Plasmonics beyond the diffraction limit," Nat. Photonics 4, 83-91 (2010).

[2] S. I. Bozhevolnyi, V. S. Volkov, E. Devaux, J.-Y. Laluet, and T. W. Ebbesen, "Channel plasmon subwavelength waveguide components including interferometers and ring resonators," Nature 440, 508-11 (2006).

[3] I. P. Radko, T. Holmgaard, Z. Han, K. Pedersen, and S. I. Bozhevolnyi, "Efficient channel-plasmon excitation by nano-mirrors," Appl. Phys. Lett. 99, 213109 (2011).

[4] C. L. C. Smith, B. Desiatov, I. Goykmann, I. Fernandez-Cuesta, U. Levy, and A. Kristensen, "Plasmonic V-groove waveguides with Bragg grating filters via nanoimprint lithography," Opt. Express 20, 5696-706 (2012).

[5] C. L. C. Smith, A. H. Thilsted, C. E. Garcia-Ortiz, I. P. Radko, R. Marie, C. Jeppesen, C. Vannahme, S. I. Bozhevnolnyi, and A. Kristensen, "Efficient Excitation of Channel Plasmons in Tailored, UV-Lithography-Defined V-Grooves," submitted (2014). 